\title{
How Effective are the Current Treatments for Children Diagnosed with Manic/Mixed Bipolar Disorder?
}

\author{
Benedetto Vitiello
}

Published online: 3 May 2013

(C) Springer International Publishing Switzerland (outside the USA) 2013

There is an ongoing debate about the diagnostic validity of bipolar disorder in children, and in particular whether chronic irritability without distinct periods of persistently elevated/expansive or angry mood, lasting at least several days, should be considered part of the bipolar phenotype [1]. The forthcoming fifth edition of the DSM (DSM-5) will add 'disruptive mood dysregulation disorder' to the psychiatric nosology, thus allowing clinicians to avoid committing to a diagnosis of bipolar disorder in the case of chronic irritability without distinct episodes of mania. While research continues to better understand the prognostic meaning of the different manifestations of extreme mood instability in childhood, severe mood instability (with or without discrete mania-like episodes lasting at least a week) constitutes a main concern in child psychiatric practice and a common cause of clinical referral and hospitalization. The search for safe and effective treatments is therefore critically important.

A number of clinical trials have tested the efficacy of medications for children diagnosed with bipolar manic/ mixed episode. Placebo-controlled studies of 3-6 weeks duration have documented the efficacy of several secondgeneration antipsychotics (SGAs), including risperidone, aripiprazole, quetiapine and olanzapine, in the acute management of manic and mixed symptoms in children (aged 10-17 years) [2]. In the USA, these four SGAs have been approved by the US FDA for paediatric use in bipolar mania or mixed states. Because of its greater potential to increase weight and induce other metabolic abnormalities,

B. Vitiello $(\bowtie)$

National Institute of Mental Health, Room 7147, 6001 Executive Blvd, Bethesda, MD 20892-9633, USA

e-mail: bvitiell@mail.nih.gov olanzapine is recommended as a second-line treatment, after considering other treatments.

The evidence base for the efficacy of non-antipsychotic mood stabilizers in children is much weaker. Lithium, valproate, carbamazepine and oxcarbazepine are used in clinical practice to stabilize severe mood instability, and a few open-label studies are suggestive of efficacy in children with bipolar disorder, but, thus far, no published placebo-controlled trial has demonstrated their efficacy [3]. Despite this, based on data in adults with bipolar disorder and children with conduct disorder, lithium has an FDAapproved indication for bipolar disorder in children aged 12 years and older.

Recently, a large, publicly funded, clinical trial has compared the effectiveness of two non-antipsychotic mood stabilizers and one SGA in paediatric mania [4]. The Treatment of Early Age Mania (TEAM) study randomized 279 children (aged 10-15 years), who were diagnosed with bipolar I disorder in a manic or mixed phase and had not been previously treated with antimanic medications, to receive risperidone, lithium or divalproex sodium for 8 weeks. Because the study was conducted in outpatient settings, severely psychotic and actively suicidal patients were excluded. However, patients with grandiose ideation consistent with mania were included and constituted the large majority of the sample. For practical reasons, the study was not masked, but the raters of the study outcomes were blinded to treatment assignment.

Treatment exposure was clearly adequate, as indicated by mean maintenance blood concentrations of $1.09 \mathrm{mEq} / \mathrm{L}$ for lithium and $113.6 \mathrm{mcg} / \mathrm{mL}$ for valproate, and by a mean titrated daily dose of $2.57 \mathrm{mg}$ of risperidone. At the end of week $8,68.5 \%$ of patients randomized to risperidone were deemed to be much or very much improved on the Clinical Global Impression for Bipolar Illness Improvement-Mania 
scale, as compared with $35.6 \%$ of those on lithium and $24.4 \%$ of those on divalproex $(p<0.001)$. The superiority of risperidone was evident across demographics and clinical characteristics, such as sex, age, severity of mania or concurrent use of stimulant medication. More than $90 \%$ of the TEAM patients met criteria for attention-deficit hyperactivity disorder (ADHD), and about one-third were receiving stable doses of stimulant concurrent with the study medication. While risperidone was more effective than lithium in patients with ADHD (regardless of stimulant treatment), no difference was found in patients without ADHD [5]. In other words, for the small group of patients whose mood symptoms were not accompanied by ADHD, lithium seemed to work as well as risperidone.

Risperidone treatment was better tolerated than lithium, as shown by a greater premature discontinuation rate on lithium $(32.2 \%)$ than risperidone $(15.7 \%, p=0.011)$. However, risperidone was accompanied by more weight gain $(3.3 \mathrm{~kg})$ than lithium $(1.42 \mathrm{~kg}, p<0.001)$ over the 8 weeks of the study, and increased blood prolactin levels [4].

Additional analyses of the TEAM database revealed that treatment effect varied significantly across the five study sites, with response rate ranging from 15.3 to $76.9 \%$. These differences could not be accounted for by patient characteristics or treatment exposure. While it is usual to find site differences in multi-site trials, their magnitude points to the influence of factors other than the pharmacological effects of specific medications on the clinical outcome of these children. Contextual factors at the family, school and community level may play an important role in the stabilization of children with severe mood dysregulation.

The results of the TEAM study should be interpreted in the context of the design and sample characteristics. The mood disorder of these children was characterized by daily rapid cycling for many years, thus indicating chronic irritability. The large majority met criteria for ADHD and oppositional defiant disorder, which, however, did not account for the extreme mood instability of these children. The superiority of an antipsychotic agent, such as risperidone, over non-antipsychotic mood stabilizers, such as lithium and valproate, in the TEAM study is consistent with the data in adults in acute mania [6]. It is also consistent with the well-documented efficacy of risperidone in alleviating symptoms of severe irritability in children with autism [7]. While the tolerability and patient/family acceptance of risperidone treatment is generally good and better than for lithium, the distal implications of the adverse metabolic effects of risperidone must be taken into account, especially when planning for long-term care. Furthermore, the long-term effects of antipsychotics on the brain remain a reason for concern when considering that their chronic use has been associated with brain tissue loss in rodents, primates and humans [8-10].
The moderator effect of ADHD in the TEAM study is intriguing but emerged from secondary analyses and was based on a small number of patients. If confirmed by future studies, it would suggest that manic-like disturbance without the features of chronic hyperactivity, impulsiveness and inattention may represent a particular subgroup of children who are responsive to a traditional antimanic agent like lithium.

Other research is in progress, and new data on the effectiveness of medications in child bipolar disorder are forthcoming. A publicly funded, multi-site, placebo-controlled trial to test the effects of lithium versus placebo in child bipolar mania will soon be completed [11]. Furthermore, as part of the TEAM study, patients who had only partially responded to lithium, risperidone or divalproex were further randomized to add one of the two other medications, and patients who had not improved on one of the three medications were randomly switched to receive one of the other two. These data will help clinicians make second-step treatment decisions in the not uncommon situations of partial response or non-response.

In summary, effective treatments for the acute management of manic symptoms in children as young as 6 years of age exist, and risperidone, an SGA, is more effective than traditional non-antipsychotic mood stabilizers. However, there are concerns about adverse effects, especially when considering long-term treatment. The search for more effective and safer agents should therefore continue.

Acknowledgments Dr. Vitiello is the chief of the Treatment and Preventive Intervention Research Branch at the National Institute of Mental Health, Bethesda, MD, USA, and adjunct professor of psychiatry at the Johns Hopkins University, Baltimore, MD, USA. The opinions and assertions contained in this article are the private views of the author and are not to be construed as official or as reflecting the views of the National Institute of Mental Health, the National Institutes of Health or the U.S. Department of Health and Human Services. Dr. Vitiello has no financial relationships with pharmaceutical companies.

\section{References}

1. Mikita N, Stringaris A. Mood dysregulation. Eur Child Adolesc Psychiatry. 2013;22(Suppl. 1):S11-6.

2. Fraguas D, Correll CU, Merchán-Naranjo J, et al. Efficacy and safety of second-generation antipsychotics in children and adolescents with psychotic and bipolar spectrum disorders: comprehensive review of prospective head-to-head and placebocontrolled comparisons. Eur Neuropsychopharmacol. 2011;21(8): 621-45.

3. Wagner KD, Redden L, Kowatch RA, et al. A double-blind, randomized, placebo-controlled trial of divalproex extended-release in the treatment of bipolar disorder in children and adolescents. J Am Acad Child Adolesc Psychiatry. 2009;48(5):519-32.

4. Geller B, Luby JL, Joshi P, et al. A randomized controlled trial of risperidone, lithium, or divalproex for initial treatment of bipolar 
I disorder, manic or mixed phase, in children and adolescents. Arch Gen Psychiatry. 2012;69(5):515-28.

5. Vitiello B, Riddle MA, Yenokyan G, et al. Moderators and predictors of treatment outcome in child bipolar disorder. J Am Acad Child Adolesc Psychiatry. 2012;51(9):867-78.

6. Cipriani A, Barbui C, Salanti G, et al. Comparative effectiveness and acceptability of antimanic drugs in acute mania: a multipletreatments meta-analysis. Lancet. 2011;378:1306-15.

7. Research Units on Pediatric Psychopharmacology Autism Network. Risperidone in children with autism and serious behavioral problems. N Engl J Med. 2002;347:314-21.

8. Vernon AC, Natesan S, Modo M, et al. Effect of chronic antipsychotic treatment on brain structure: a serial magnetic resonance imaging study with ex vivo and postmortem confirmation. Biol Psychiatry. 2011;69(10):936-44.

9. Dorph-Petersen KA, Pierri JN, Perel JM, et al. The influence of chronic exposure to antipsychotic medications on brain size before and after tissue fixation: a comparison of haloperidol and olanzapine in macaque monkeys. Neuropsychopharmacology. 2005;30(9):1649-61.

10. Ho BC, Andreasen NC, Ziebell S, et al. Long-term antipsychotic treatment and brain volumes: a longitudinal study of first-episode schizophrenia. Arch Gen Psychiatry. 2011;68(2):128-37.

11. Findling RL, Frazier JA, Kafantaris V, et al. The Collaborative Lithium Trials (CoLT): specific aims, methods, and implementation. Child Adolesc Psychiatry Ment Health. 2008;2(1):21. 